\section{1/f Macroscopic Quantum Fluctuations of Elec- tric Currents Due to Bremsstrahlung with Infra- red Radiative Corrections}

\author{
Peter H. Handel \\ Department of Physics, University of Missouri-St. Louis, \\ St. Louis, Missouri 63121 \\ (Z. Naturforsch. 30 a, 1201-1202 [1975] ; \\ received March 17, 1975)
}

Due to interaction with the quantized electromagnetic field, any current carrier involved in some scattering process may emit low frequency photons. This photon emission carries negligible energy but modulates the current nevertheless, resulting in $1 / f$ and $1 / \Delta f$ noise.

In electronics, electrical engineering, and telecommunications $1 / f$ noise is well known experimentally as the general form of low frequency electrical fluctuations, but no satisfactory and sufficiently general interpretation of this phenomenon has been given so far.

Consider first the wave function $\psi$ of a beam of charged particles, e.g. electrons, which have been scattered by an arbitrary potential. This wave function $\psi$ can be written in terms of the corresponding scattering matrix elements for elastic scattering and for scattering with bremsstrahlung. Including the infrared radiative corrections ${ }^{1}$ in the coherent states representation ${ }^{2}$ and using the propagator formalism, we construct the wave function in the form $(\hbar=c=1)$

$$
\begin{aligned}
& \psi=a \exp \{i(\boldsymbol{p} \boldsymbol{r}-E t)\}+\int_{\varepsilon_{0}}^{\varepsilon_{1}} b_{\varepsilon} \exp \left\{i \boldsymbol{p}^{\prime} \boldsymbol{r}-i E t\right\} \\
& \cdot\left(e^{i \varepsilon t} a_{\varepsilon}^{+}+e^{-i \varepsilon t} a_{\varepsilon}\right)(\mathrm{d} \varepsilon / \sqrt{\varepsilon})
\end{aligned}
$$

where the coefficients of the elastic part $(a)$ and of the bremsstrahlung part $\left(b_{\varepsilon}\right)$ satisfy the relation

$$
b_{\varepsilon} / a=(\alpha A)^{1 / 2}\left(\varepsilon / \varepsilon_{0}\right)^{\alpha A / 2} \equiv \varrho_{\varepsilon} .
$$

The appropriate phase factor $e^{i \gamma(\varepsilon)}$ has been omitted in Eq. (2) and is included in the definition of $a_{\varepsilon}{ }^{+}$ as a normed superposition of photon creation oper- ators

$$
\begin{gathered}
a_{\varepsilon}^{+}=\varepsilon^{-1} \int_{4 \pi} f_{\varepsilon}^{*}(\theta, \varphi) a_{\boldsymbol{k}}^{+} k^{2} \mathrm{~d} \Omega_{\boldsymbol{k}} ; \\
{\left[a_{\varepsilon}, a_{\varepsilon^{\prime}}^{+}\right]=\delta\left(\varepsilon-\varepsilon^{\prime}\right),} \\
\int_{4 . \tau}\left|f_{\varepsilon}(\theta, \varphi)\right|^{2} \mathrm{~d} \Omega_{\boldsymbol{k}}=1, \quad k \equiv|\boldsymbol{k}|=\varepsilon .
\end{gathered}
$$

In Eq. (1) $\varepsilon$ represents the radiative energy loss of the electron, $\varepsilon_{0}$ is the detection threshold, i. e. the lowest frequency of the $1 / f$ noise measurement, and $\varepsilon_{1}$ is the highest possible energy loss, which is the energy of the incoming electrons $E$. However, Eq. (1) with Eq. (3) is exact only for values of $\varepsilon \ll E$ which are low enough, so that the small momentum variation caused by the energy loss $\varepsilon$ is negligible and the prime can be droped in Equation (1). Note that $\boldsymbol{p}^{\prime}-\boldsymbol{p}$ is not the momentum change in the basic scattering process which induces soft photon emission, but rather the small fluctuation of this momentum transfer due to the energy difference $\varepsilon$ introduced by the emission. Writing $\boldsymbol{r} \cdot \boldsymbol{p}=\varepsilon r / v=\varepsilon \Delta t$, we have to limit ourselves to frequencies $\varepsilon$ lower than the reciprocal flight time of the electrons through the measuring area. For higher frequencies Eq. (3) has to be modified, but these higher frequencies do not yield significant contributions due to the energy denominators.

In Eq. (2) $\alpha$ is the fine structure constant $137^{-1}$ and $\alpha A$ is the coefficient of $\ln (\varepsilon / E)$ in the expression of the infrared exponent ${ }^{3}$ for small $4-$ momentum transfers $\left(\left|q^{2}\right| \ll m^{2}\right)$ :

$$
\begin{aligned}
2 \alpha(B+\tilde{B}) & \cong \alpha A \ln \frac{\varepsilon}{E}=-\frac{2 \alpha q^{2}}{3 \pi m^{2}} \ln \frac{\varepsilon}{E} \\
& \simeq \frac{8 \alpha}{3 \pi} \beta^{2}\left(\sin ^{2} \frac{\theta_{0}}{2}\right) \ln \frac{\varepsilon}{E} .
\end{aligned}
$$

The last form is a nonrelativistic approximation $(\beta=v / c \ll 1)$ for small $\varepsilon\left(\beta^{\prime}=\beta\right)$, and $\theta_{0}$ is the scattering angle in the basic scattering process.

The current corresponding to Eq. (1) is

$$
\begin{aligned}
\boldsymbol{j}=\frac{-\boldsymbol{i}}{m} \psi_{\nabla}^{+} \psi & =\boldsymbol{p} \frac{|\boldsymbol{a}|^{2}}{m}\left\{1+2 \int_{\varepsilon_{0}}^{\varepsilon_{1}} \varrho_{\varepsilon}\left(e^{i \varepsilon t} a_{\varepsilon}^{+}+e^{-i \varepsilon t} a_{\varepsilon}\right) \frac{\mathrm{d} \varepsilon}{\sqrt{\varepsilon}}\right. \\
& \left.+\int_{\varepsilon_{0}}^{\varepsilon_{1}} \int_{\varepsilon_{0}}^{\varepsilon_{1}} \varrho_{\varepsilon} \varrho_{\varepsilon^{\prime}}\left[e^{i\left(\varepsilon^{\prime}-\varepsilon\right) t} a_{\varepsilon} a_{\varepsilon^{\prime}}^{+}+e^{-i\left(\varepsilon+\varepsilon^{\prime}\right) t} a_{\varepsilon} a_{\varepsilon^{\prime}}+e^{i\left(\varepsilon+\varepsilon^{\prime}\right) t} a_{\varepsilon}^{+} a_{\varepsilon^{\prime}}{ }^{+}+e^{i\left(\varepsilon-\varepsilon^{\prime}\right) t} a_{\varepsilon}^{+} a_{\varepsilon^{\prime}}\right] \frac{\mathrm{d} \varepsilon}{\sqrt{\varepsilon}} \frac{\mathrm{d} \varepsilon^{\prime}}{\sqrt{\varepsilon^{\prime}}}\right\} .
\end{aligned}
$$

The vacuum expectation value of this current is proportional to the cross section ${ }^{1}$, as required for consistency :

$$
\langle\boldsymbol{j}\rangle=\boldsymbol{p} \frac{|\boldsymbol{a}|^{2}}{m}\left[1+\int_{\varepsilon_{0}}^{\varepsilon_{1}} \varrho_{\varepsilon}^{2} \frac{\mathrm{d} \varepsilon}{\varepsilon}\right] .
$$

The corresponding autocorrelation function is de- fined up to order $\varrho^{2}$ as

$$
\begin{aligned}
& \frac{1}{2}\left\langle\delta \boldsymbol{j}^{+}(t) \delta \boldsymbol{j}(t+\tau)\right\rangle \\
& \quad+\text { c. c. }=4 \boldsymbol{p}^{2} \frac{|a|^{4}}{m^{2}} \int_{\varepsilon_{0}}^{\varepsilon_{1}} \varrho_{\varepsilon}^{2} \cos (\varepsilon \tau) \frac{\mathrm{d} \varepsilon}{\varepsilon},
\end{aligned}
$$

where c. c. is the complex conjugate and $\delta \boldsymbol{j}=\boldsymbol{j}-\langle\boldsymbol{j}\rangle$ is the current fluctuation. If we apply the Wiener- 
Khintchine theorem and restore the units, we obtain the spectral density of current noise $(\varepsilon=h f)$

$$
\begin{aligned}
\left\langle(\delta \boldsymbol{j})^{2}\right\rangle_{f}\langle\boldsymbol{j}\rangle^{-2} & =\frac{4 \varrho_{f}^{2} f^{-1}}{\left[1+\int_{f_{0}}^{f_{1}} \varrho_{\mathrm{f}^{\prime}}{ }^{2} \mathrm{~d} f^{\prime} / f^{\prime}\right]^{2}} \\
& =4 \alpha A\left(\frac{f f_{0}}{f_{1}{ }^{2}}\right)^{\alpha .4} f^{-1} \approx 4 \alpha A f^{-1} .
\end{aligned}
$$

The maximal value of $\alpha A$ allowed by Eq. (2) is $8 \alpha / 3 \pi \approx 161^{-1}$ which corresponds to the reflection of a normally incident ultrarelativistic beam of charged particles of arbitrary nature in vacuum. In this case the $1 / f$ noise coefficient $\alpha A$ exceeds the experimental ${ }^{4}$ value of $10^{-3}$ obtained by Hooge from various measurements by a factor of 6.2. The factor $\left(f f_{0} / f_{1}^{2}\right)^{\alpha A}$ is close to unity for practical measurements.

* Work supported by the National Science Foundation.

1 D. R. Yennie, S. C. Frautschi, and H. Suura, Ann. Physics New York 13, 379 [1961], Eqs. (2.59) - (2.60) with $G_{1}=0, \Delta E=\varepsilon_{1}$ and the integral broken up at $\varepsilon_{0}$.
In the case of alternating current of frequency $\omega_{0} /(2 \pi)$ pure states of the form

$$
\begin{gathered}
\psi=a\left[\exp \left\{i\left(\boldsymbol{p}_{1} \cdot \boldsymbol{r}-E_{1} t\right\}+\exp \left\{i\left(\boldsymbol{p}_{2} \cdot \boldsymbol{r}-E_{2} t\right)\right\}\right]\right. \\
+\int_{\varepsilon_{0}}^{\varepsilon_{1}} b_{\varepsilon}\left[\exp \left\{i\left(\boldsymbol{p}_{1}{ }^{\prime} \cdot \boldsymbol{r}-E_{1} t\right)\right\}+\exp \left\{i\left(\boldsymbol{p}_{2}{ }^{\prime} \cdot \boldsymbol{r}-E_{2} t\right)\right\}\right] \\
\cdot\left(e^{-i \varepsilon t} a_{\varepsilon}{ }^{+}+e^{-i \varepsilon t} a_{\varepsilon}\right) \frac{\mathrm{d} \varepsilon}{\sqrt{\varepsilon}}
\end{gathered}
$$

with $\omega_{0}=E_{1}-E_{2}$ are considered instead of Eq. (1), with the phase factor $e^{i \gamma(\varepsilon)}$ retained explicity in Equation (2). Calculating the current in the same way and performing a time average which is denoted by a bar, we obtain the spectral density

${\overline{\left\langle\boldsymbol{j}^{+}(t) \boldsymbol{j}(t)\right\rangle_{\omega_{0}-\varepsilon}}}=\left(|a|^{4} / 2 m^{2}\right)\left(\boldsymbol{p}_{1}+\boldsymbol{p}_{2}\right)^{2} \varrho_{\varepsilon}{ }^{2} \varepsilon^{-1}(11)$ in the lower sideband, and

${\overline{\left\langle\boldsymbol{j}^{+}(t) \boldsymbol{j}(t)\right\rangle_{\omega_{0}+\varepsilon}}}=\left(|a|^{4} / 2 m^{2}\right)\left(\boldsymbol{p}_{1}+\boldsymbol{p}_{2}\right)^{2} \varrho_{\varepsilon}{ }^{2} \varepsilon^{-1}(12)$ in the upper sideband. This is $1 / \Delta f$ noise.

2 V. Chung, Phys. Rev. 140 B, 1110 [1965].

${ }^{3}$ See Ref. ${ }^{1}$ Eq. (2.34).

${ }^{4}$ F. N. Hooge, Physica 60, 130 [1972]. 